\title{
Adjacent Order Separation for Variable Speed Bearing with Peak Detection and Fixed-point ICA
}

\author{
Bin Chen ${ }^{\mathrm{a}}{ }^{*}$, Mi Liu ${ }^{\mathrm{b}}$, Yuan Zhou ${ }^{\mathrm{c}}$ and Baocheng Gao ${ }^{\mathrm{d}}$ \\ School of Automation, Beijing University of Posts and Telecommunications, Beijing 100876, China.

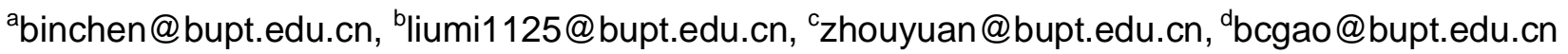 \\ * Corresponding author: Bin Chen; E-mail: binchen@bupt.edu.cn
}

\begin{abstract}
Keywords: adjacent order, wheel-bearing, independent component analysis, peak detection Abstract. To solve the problem of adjacent order overlap for variable speed bearings, a novel method with the fixed-point independent component analysis (ICA) and peak detection is proposed. Firstly, the mixed vibration signal is whitened by through of the singular value decomposition. Then it introduces fixed-point ICA technique to separate the mixed vibration signal into different independent components. To improve the performance of separation, the adjacent orders are extracted by peak detection according to the energy distribution characteristics of separated signal. The effectiveness of proposed method is verified. Experimental results show that proposed method provides better performance on adjacent order separation for roller bearings.
\end{abstract}

\section{Introduction}

Wheel-bearing is one of the most important mechanical parts of the train. To guarantee the safety, reliability and efficiency, the detection of incipient defects of wheel-bearing is necessary and great important. The vibration-based diagnostic method is an effective way to defect detection. Some researchers have given more study and developed a variety of techniques [1]. Envelope analysis is one of the most popular techniques for rolling element bearing (REB). Its main idea is to detect the characteristic frequencies corresponding to localized faults of REB [2]. Other processing techniques, such as empirical mode decomposition (EMD) [3, 4], stochastic resonance [5], and wavelet analysis have also been applied to the bearing fault detection in recent years.

Among those methods, order tracking [6] is an effective approach and has been used to the REB diagnosis. Different from traditional frequency-based techniques, order tracking resamples the vibration signal at constant angular increments of a reference shaft. In this way, it could avoid spectral smearing due to speed variation, and remove the background noise by through of synchronous averaging. In addition, this method requires a tachometer or an encoder to provide a phase reference signal. It not only increases the measurement cost, but also brings inconvenience in installation. To overcome the weakness, some tacholess order tracking techniques have been developed [9]. The tacholess order tracking directly extracts the shaft speed information from vibration signal by through of some signal processing techniques, such as short-time Fourier transform (STFT). However, the adjacent orders in order spectrum may be overlapped, which has great influences on diagnosis accuracy.

The independent components analysis (ICA) is based on high order statistical characteristic analysis. This technique could find underlying components from multidimensional statistical information, and transform multichannel signals into independent source signals. It has become a powerful tool for array signal processing, and widely used in different applications [7]. The Fast-ICA algorithm belongs to the family of fix-point algorithms for ICA [8], which is based on the iteration to search for the maximum non-Gaussian component. The observed vibration signal of REB can be considered as the superposition of a number of independent signal sources. To speed up the calculation, this paper employs the fixed-point ICA (FICA) to separate the mixed vibration signal. To further eliminate other small residual component, the peak detection (PD) is also introduced to the adjacent order separation of the REB.

The paper is arranged as follows. Section 2 gives the principle of order separation with fixed-point ICA and peak detection (FICA-PD). Proposed method is verified by simulation and 
experiment in section 3. Conclusions are summarized in section 4.

\section{Order Separation with FICA-PD}

Basic ICA Model. Given a set of observable random variables $\mathbf{X}\left(\mathbf{X}=\left[x_{1}(t) x_{2}(t) \mathrm{K} x_{n}(t)\right]\right)$ and sources $\mathbf{S}=\left[s_{1}(t) s_{2}(t) \mathrm{K} s_{n}(t)\right]$, the basic linear mixture of ICA model can be described as follows.

$$
\left[x_{1}(t) x_{2}(t) \mathbf{K} x_{n}(t)\right]^{T}=\mathbf{A}\left[s_{1}(t) s_{2}(t) \mathbf{K} s_{n}(t)\right]^{T}
$$

where $t$ is the time or the sample index, $\mathbf{A}$ is unknown mixed matrix, $T$ stands for the transpose of a matrix. In ICA model, sources are supposed as statistical independent, and have the non-Gaussian distribution. The mixed matrix A can be estimated according to the prior probability distribution of sources.

Then, the separated matrix could be obtained according to the inverse of mixed matrix.

$$
\mathbf{W}=\mathbf{A}^{-1}
$$

Finally, the independent components can be calculated as follows.

$$
\mathbf{S}=\mathbf{W} * \mathbf{X}
$$

FICA-PF Algorithm Design. To separate the adjacent order components in order spectrum, the mixed vibration signals are preprocessed. Firstly, let observable data have the mean of zero by through of following formula.

$$
\mathbf{X}^{\prime}=\mathbf{X}-E(\mathbf{X})
$$

where function $E(\square)$ denotes the mean of data. According to Eq.(3), the mean of variable $\mathbf{S}$ is also zero. As we know, the components in observable vector should be uncorrelated in ICA. The preprocessed data are then whitened by through of the singular value decomposition. Finally, the input data has characteristics of zero mean, uncorrelated, and unit variance. Therefore, the mixed matrix becomes orthogonal.

After data preprocessing, the key problem is how to find the better separated matrix. The detailed steps are as follows.

Step 1: Choose an initial random vector wi(0) that satisfies $\left\|_{w_{i}}(0)\right\|_{2}=1$, where $\left\|_{\mathrm{w}}\right\|_{2}$ is 2-norm of w.

Step 2: According to the central limit theorem, if the random variable $Z$ is composed of independent random variables, as long as with finite mean and variance, it is closer to Gaussian distribution. Therefore, the measure of non-Gaussianity could be used to represent the independence between separated results. If it reaches the maximum, the separation process is finished. In this paper, the negative entropy is used to measure the non-Gaussianity.

$$
N_{g}(Z)=E(g(z))-E\left(g\left(Z_{\text {Gauss }}\right)\right)^{2}
$$

Where $g(\square)$ is the chosen nonlinear function, for example $g_{3}(\mathrm{y})=y e^{-y^{2} / 2}$.

The learning rule is to find a direction that makes the estimated order has maximum non-Gaussianity. To simply the matrix inversion, apply with the following simplified iteration formula in this paper.

$$
\mathbf{w}(\mathrm{k}+1)=E\left[Z \square g\left(\mathbf{w}^{\prime}(\mathrm{k}) Z\right)\right]-E\left[\bar{g}\left(\mathbf{w}^{\prime}(\mathrm{k}) Z\right)\right] \mathbf{w}(\mathrm{k})
$$

Where $\bar{g}(\square)$ is the derivative of function $g(\square)$.

Step 3: Normalized the data.

$$
w(\mathrm{k}+1) \leftarrow w(\mathrm{k}+1) /\|\mathrm{w}(\mathrm{k}+1)\|_{2}
$$

If it does not satisfy $\mathbf{w}(k+1)-\mathbf{w}(k) \leq \square$, where $\square$ is a convergence parameter, then go back to the step 2. Finally, the separated matrix could be obtained.

To further eliminate the small residual components, the peak detection is introduced to separate the adjacent orders in order spectrum for REB. Firstly, divide separated signal into segments. The spectrum of the segment with STFT is as following. 


$$
F(t, f)=\int_{-\infty}^{+\infty}\left[x(\tau) \gamma^{*}(\tau-t)\right] \exp (-j 2 \pi f \tau) d \tau
$$

where * denotes complex conjugate, $\gamma(t)$ is time window function. The corresponded formula for calculating the discrete signal is defined as follows.

(9)

$$
F(n, k)=\sum_{i=0}^{N-1}\left[x(i) \gamma^{*}(i-n)\right] \exp \left(-j \frac{2 \pi k i}{N}\right)
$$

Where $\mathrm{N}$ is the length of signal, $\mathrm{n}, \mathrm{k}$ are the discrete time and frequency in STFT spectrum.

Finally, find and record the maximum peak from STFT spectrum of each segment. The interest component with the largest energy could be separated.

\section{Simulation and Experiment}

To verify the validity of proposed algorithm, the following simulation and experiment are made.

Simulation. An acceleration process of roller bearing is simulated. Four components corresponding to the roller, outer race, inner race and cafe of REB are simulated and linear mixed, as shown in Fig.1. The fault characteristic frequencies changes from $0 \mathrm{~Hz}$ to the end of $6,10,14,20 \mathrm{~Hz}$ during 50 seconds, respectively. The amplitude of each component is set to 1 .

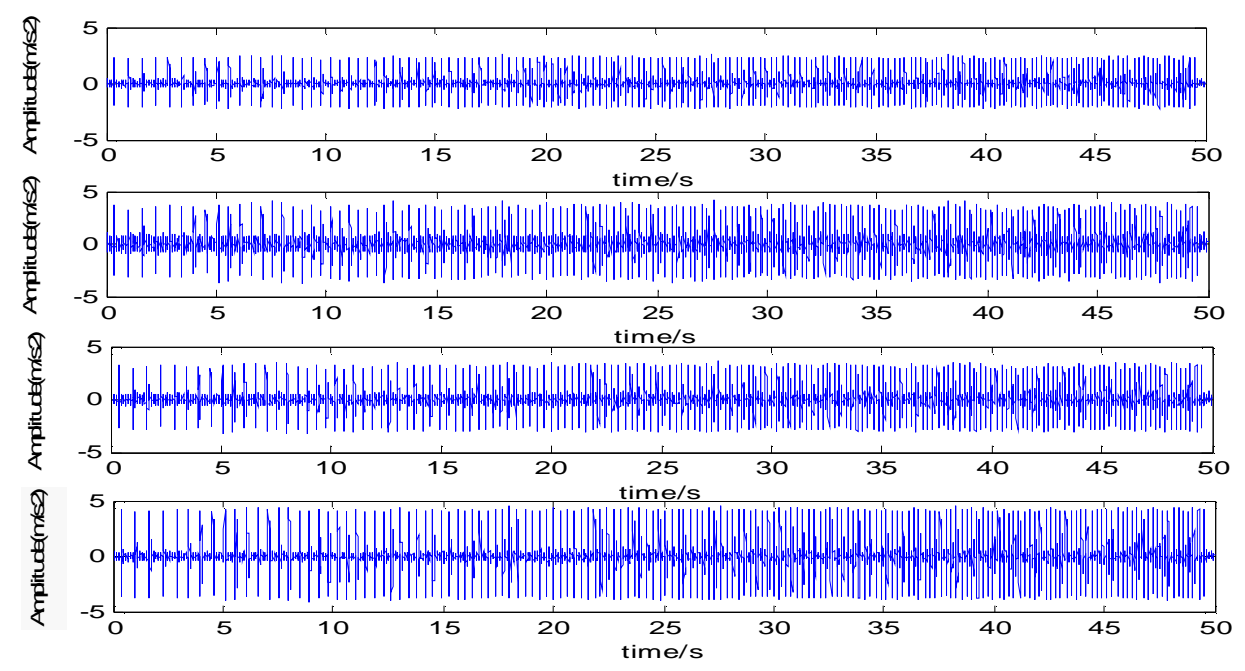

Fig. 1 Mixed signals

Apply with the proposed Fast-ICA algorithm to process the mixed vibration signals, the results are show in Fig.2. We can see the different characteristic frequencies are separated obviously from $5 \mathrm{~s}$ to $50 \mathrm{~s}$. While in process of startup, the adjacent components get very closer to each other, and could not be separated completely. The main reason is that the theoretical interval for different components in low speed condition is small. Therefore, they become smearing. 


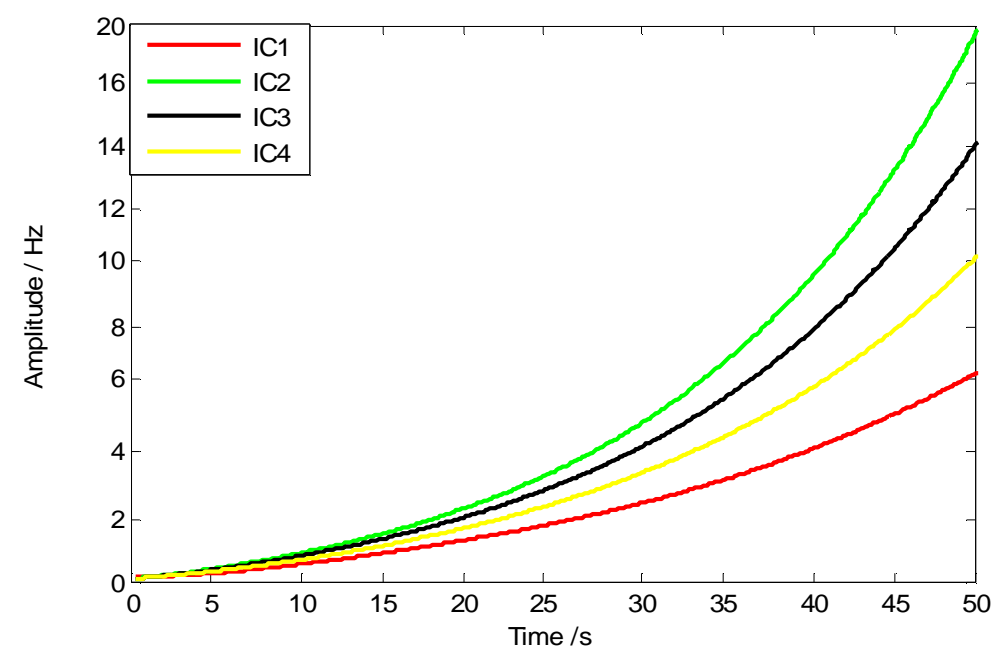

Fig. 2 Extracted frequency component with FICA-PF

Experiment. In this section, a dataset collected from test-rig is used to further illustrate the effectiveness of the proposed method. Fig. 3 shows the structure of test rig, consisting of rotor, frequency converter, bearing and gearbox.

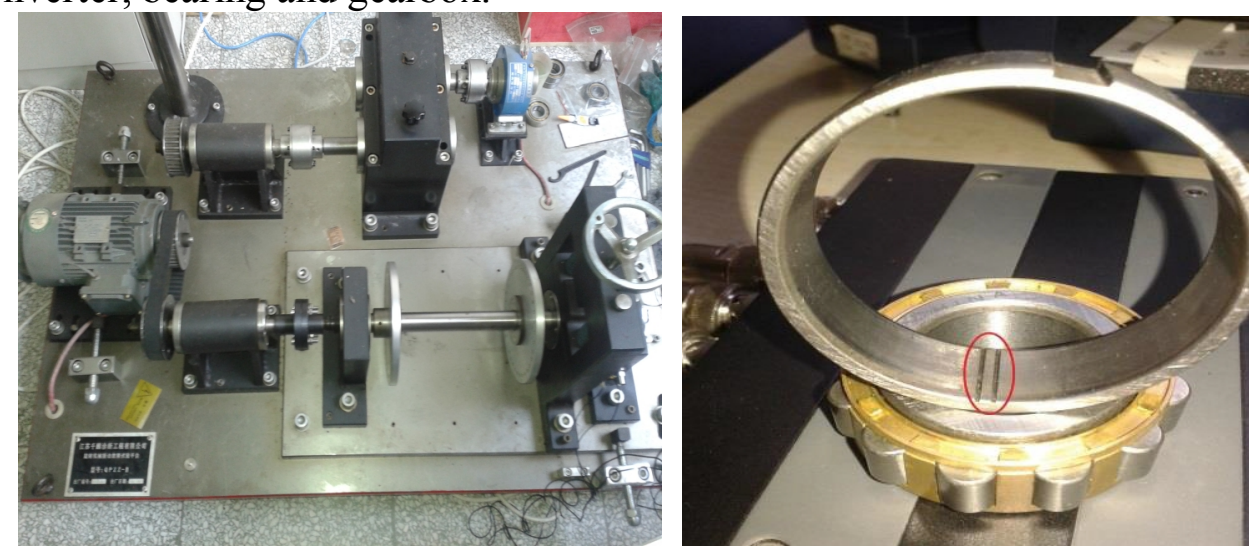

Fig. 3 Test rig and the outer race fault bearing

The vibration signals are collected through accelerometers using B\&K PULSE at sampling frequency $32768 \mathrm{~Hz}$. In addition, four accelerometers are mounted on the surface of outer race at 3, 6, 9 and 12 o'clock. The type of experimental fault bearing is N205. The roller diameter $d$ and pitch diameter D are $13 \mathrm{~mm}$ and $38 \mathrm{~mm}$. In addition, the number of rollers is 10, and the contact angle equals to zero. In experiment, the speed of shaft changes from $200 \mathrm{rpm}$ to $1200 \mathrm{rpm}$ during $50 \mathrm{~s}$. The waveform of one second vibration signals are show in Fig.4.

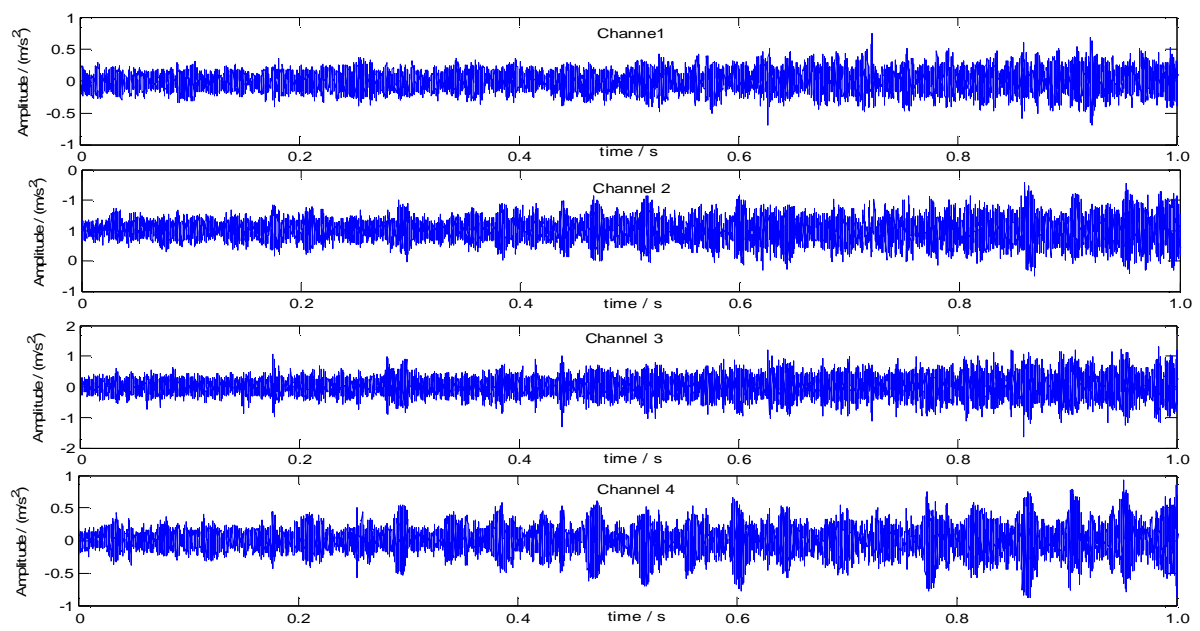

Fig. 4 The waveforms of vibration signal (4-Channels)

Fig.5 shows the results of order spectrum after preprocessing with proposed FICA-PF 
algorithm.

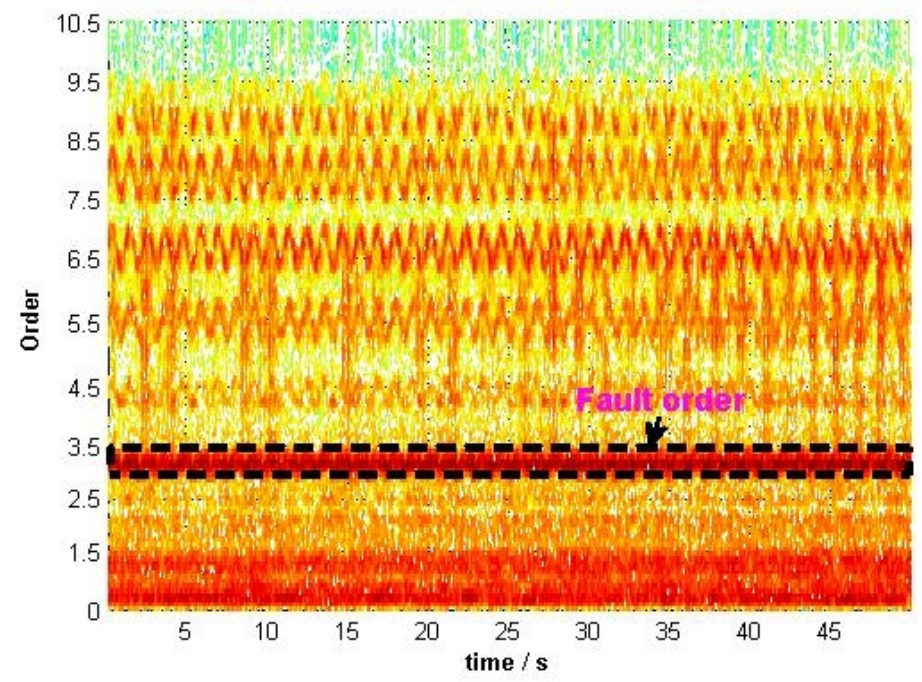

Fig. 5 The order spectrum

We can see the fault order component could be separated from variable speed vibration signal. Moreover, they are fluctuating near the theoretical value $(3.3 \mathrm{~Hz})$, showing a narrow band. The theoretical fault order is calculated as follows.

$$
\text { Order }=\frac{1}{2} n\left(1-\frac{\mathrm{d}}{D} \cos \alpha\right)=\frac{1}{2} * 10 *\left(1-\frac{13}{38} * \cos 0\right) \approx 3.3 \mathrm{~Hz}
$$

Compared to the simulated results, there are some residual components in order spectrum. The reason is that there are some random noises in real vibration signal. In addition, there are some fundamental frequency and its harmonics of the shaft because of misalignment or imbalance.

\section{Conclusions}

A novel method with the fixed-point independent component analysis and peak detection is proposed to extract the order components in variable speed condition. By preprocessing the vibration signal with singular value decomposition, the mixed matrix becomes orthogonal. The Fast-ICA could separate the mixed orders into different independent components effectively. The peak detection technique eliminates small residual components. Simulation and experiment results show that proposed method has abilities of adjacent order separation.

\section{Acknowledgments}

This work is jointly supported by the National Natural Science Foundation of China (Grant No. 11304019) and the Fundamental Research Funds for the Central Universities (No.2014RC0401).

\section{References}

[1] R.B. Randall, J. Antoni. Rolling element bearing diagnostics-A tutorial. Mechanical Systems and Signal Processing, 2011, 25:485-520.

[2] K.R. Fyfe, E.D.S. Munck. Analysis of computer order tracking. Mechanical Systems and Signal Processing, 1997,11( 2) :187-205

[3]Yu Guo, Jing Na, Bin Li, Rong-Fong Fung. Envelope extraction based dimension reduction for independent component analysis in fault diagnosis of rolling element bearing. Journal of Sound and Vibration, 2014, 333(13): 2983-2994.

[4] D. Yu, J. Cheng, Y. Yang. Application of EMD method and Hilbert spectrum to the fault diagnosis of roller bearings. Mechanical Systems and Signal Processing, 2005, 19:259-270. 
[5] W. Guo, P.W. Tse, A. Djordjevich. Faulty bearing signal recovery from large noise using a hybrid method based on spectral kurtosis and ensemble empirical mode decomposition. Measurement, 2012, 45:1308-1322.

[6] J. Tan, X. Chen, J. Wang, H. Chen, et al. Study of frequency-shifted and re-scaling stochastic resonance and its application to fault diagnosis. Mechanical Systems and Signal Processing, 2009, 23:811-822.

[7] A.J. Bell, J. Sejnowsket. An information maximization approach to blind separation and blind deconvolution. Neural Computation, 1995, 7:1129-1132.

[8] A. Hyväarinen. A family of fixed-point algorithms for independent component analysis. Proceedings of IEEE International conference on Acoustics, Speech and Signal Processing, Munich, Germany, 1997, 3917-3920.

[9] M. Zhao, J. Lin, X.Q. Xu, Y.G. Lei. Tacholess envelope order analysis and its application to fault detection of rolling element bearings with varying speeds. Sensors, 2013, 13:10856-10875. 